\title{
The effects of polyphenol-rich chokeberry juice on fatty acid profiles and lipid peroxidation of active handball players: results from a randomized, double blind, placebo controlled study
}

\begin{tabular}{|c|c|}
\hline Journal: & Canadian Journal of Physiology and Pharmacology \\
\hline Manuscript ID & cjpp-2015-0575.R1 \\
\hline Manuscript Type: & Article \\
\hline Date Submitted by the Author: & 26-Feb-2016 \\
\hline Complete List of Authors: & $\begin{array}{l}\text { Petrovic, Snjezana; Institute for Medical Research, University of Belgrade, } \\
\text { Serbia, Centre of Research Excellence in Nutrition and Metabolism } \\
\text { Arsic, Aleksandra; Centre of Research Excellence in Nutrition and } \\
\text { Metabolism, Institute for Medical Research, University of Belgrade, Serbia } \\
\text { Glibetic, Marija; Institute for Medical Research, University of Belgrade, } \\
\text { Serbia, Centre of Research Excellence in Nutrition and Metabolism } \\
\text { Cikiriz, Nikola; Institute of Hygiene, Military Medical Academy, Belgrade, } \\
\text { Serbia, Department of Exercise Physiology, } \\
\text { Jakovljevic, Vladimir; Facuklty of Medical Sciences, University of } \\
\text { Kragujevac, Department of Physiology } \\
\text { Vucic, Vesna; Institute for Medical Research, University of Belgrade, } \\
\text { Serbia, Centre of Research Excellence in Nutrition and Metabolism }\end{array}$ \\
\hline Keyword: & handball, chokeberry juice, Aronia melanocarpa, polyphenols, fatty acids, \\
\hline
\end{tabular}


1 The effects of polyphenol-rich chokeberry juice on fatty acid profiles and lipid peroxidation

2 of active handball players: results from a randomized, double blind, placebo controlled study

3

4 Snjezana Petrovic ${ }^{\mathrm{a}}$, Aleksandra Arsic ${ }^{\mathrm{a}}$, Marija Glibetic ${ }^{\mathrm{a}}$, Nikola Cikiriz ${ }^{\mathrm{b}}$, Vladimir $5 \quad$ Jakovljevic $^{\mathrm{c}}$, Vesna Vucic ${ }^{\mathrm{a}}$

$6{ }^{\mathrm{a} C}$ Centre of Research Excellence in Nutrition and Metabolism, Institute for Medical Research,

7 University of Belgrade, Serbia

$8{ }^{\mathrm{b}}$ Department of Exercise Physiology, Institute of Hygiene, Military Medical Academy,

9 Belgrade, Serbia

$10{ }^{\mathrm{c} D e p a r t m e n t}$ of Physiology, Faculty of Medical Sciences, University of Kragujevac, Serbia

12 Corresponding author:

13 Dr Vesna Vucic

14 Centre of research excellence in nutrition and metabolism

15 Institute for Medical Research

16 University of Belgrade

17 Tadeusa Koscuska 1

18 PO Box 102

1911129 Belgrade

20 Serbia

21 E-mail address: vesna.vucic.imr@gmail.com

22 Tel: $+38111303-1997$

23 Fax: +381 11 2030-169 
Abstract

The effect of polyphenol-rich chokeberry juice consumption on plasma phospholipids fatty acid profiles of 32 active male and female handball players was examined. This randomised double-blind, placebo controlled study was conducted during the preparatory training in a closed campus, where 18 players $(8 \mathrm{M}, 10 \mathrm{~F})$ consumed $100 \mathrm{ml}$ of chokeberry juice, while 14 players $(7 \mathrm{M}, 7 \mathrm{~F})$ consumed placebo. Lipid status, glucose, thiobarbituric acid reactive substances (TBARS) and percentages of fatty acids were assessed at baseline and the end of the study. Consumption of chokeberry juice induced decreases of C18:1n-9 and C18:3n-3 in men, but no changes in female players. However, placebo controlled groups had reduced proportions of mono- (C16:1n-7, C18:1n-7), and polyunsaturated fatty acids (PUFAs: $\mathrm{C} 18: 3 n-3, \mathrm{C} 20: 5 n-3$, and $\mathrm{C} 22: 4 \mathrm{n}-6$ ) in males, as well as n-6 PUFAs and total PUFAs in females after consumption. These results indicate that chokeberry juice had a weak impact on attenuating the effect of intensive training in active handball players.

Keywords: handball, chokeberry juice, Aronia melanocarpa, polyphenols, fatty acids, placebo, randomized controlled trial

\section{(1)}




\section{Introduction}

50

51

52

Many athletes use various dietary supplements to improve their performances and to protect their health from adverse effects of prolonged strenuous training. However, the effectiveness and benefits of such supplementation are often questionable. It has been shown that athletes under a regular training program exhibit a substantial increase in oxidative stress than healthy sedentary people (Lafay et al. 2009). Although small amounts of reactive oxygen species (ROS) are constantly being generated in cells during normal physiological processes and even participate in different cell functions and immunity, overproduction of ROS causes oxidative damages of lipids, proteins and DNA, chronic inflammation and is involved in many acute and chronic diseases. The membrane phospholipids, especially polyunsaturated fatty acids (PUFA) are prone to oxidative damage (Solans et al. 2000), so increased oxidative stress and altered fatty acid (FA) status in plasma and erythrocytes phospholipids are commonly found in elite athletes (Arsic et al. 2012, 2015; Tepsic et al. 2009, 2011). Accordingly, administration of different antioxidants, such as selenium, vitamin $\mathrm{E}$, vitamin $\mathrm{C}$ or polyphenols is rather common and revealed that it was possible to improve exercise-induced antioxidant response even in already adopted antioxidant status (Margaritis et al. 2003; Morillas-Ruiz et al. 2005, 2006).

Berries are important dietary sources of polyphenols, in particular anthocyanins, which are well known for their strong antioxidant activity (Szajdek and Borowska 2008). Among berries, chokeberry (Aronia melanocarpa L.) showed the highest contents of polyphenols and thus antioxidant capacity (Bermudez-Soto and Tomas-Barberan 2004; Zheng and Wang 2003). The most abundant polyphenols in chokeberry are procyanidins, anthocyanins and phenolic acids. These polyphenols are largely responsible for many of the chokeberry medicinal properties and physiological effects, given that their high content and distribution 
pattern remain almost the same in juice and pomace, as in fresh berries (Kulling and Rawel 2008). A plenty of evidences arising from in vitro, animal and human studies reported that chokeberry juice attenuates hyperglycemia and hypertriglyceridemia and reduces systolic and diastolic blood pressure, as well as total cholesterol and LDL in serum, while increases level of HDL2 cholesterol in hyperlipidemic people (Broncel et al. 2010; Skoczynska et al. 2007). Beneficial effects of chokeberry juice on cellular antioxidant enzymes and erythrocyte membrane FA status exerted through increased n-3 PUFA, total PUFA and average degree of membrane phospholipids FA unsaturation in healthy women were also reported (Kardum et al. 2014a). However, the effects of polyphenol rich chokeberry juice on FA status in elite athletes have not been investigated so far.

As a strenuous intermittent sport, handball places an important stress on a players aerobic and anaerobic metabolism and produces a marked state of oxidative stress (Marin et al. 2011). Plasma FA status of handball players is also altered (Mougios et al. 1995). Taking into account all these facts, this study was aimed to estimate the influence of four weeks regular consumption of chokeberry juice on body composition and FA profile of plasma phospholipids in young elite male and female handball players.

\section{Material and methods}

\subsection{Subjects}

The subjects were recruited from elite handball clubs from Belgrade and Kragujevac, Serbia. The study included 15 male handball players, aged 16-20 years (18.5 \pm 1.06 years), playing for the Junior National Selection Team, and 17 female handball players, 16-19 years of age (17.2 \pm 0.93 years). All study participants were apparently healthy, had no active sports injuries, did not use any medications or oral contraceptives, and were non-smokers. Standardized questionnaires conducted under the supervision of a trained nutritionist were 
used to collect general data, nutritional habits and use of dietary supplements. The athletes who used any dietary supplements at least a month before the study, were excluded. All female study participants reported regular menstrual cycles (26-32 days). All participants, (or their parents if they were under the age of 18) signed an informed consent document. The study was approved by the Ethical committee of Faculty of Medical Sciences, University of Kragujevac.

\subsection{Study design}

The study was conducted after regular competition season, during the period of preparatory training prior to the next competition season, and lasted for four weeks. The participants were settled in a closed campus in Serbia, all having the same training and nutritional regime, which excluded intake of berries. Regular training regimen before the study included combination of aerobic, conditioning and strength exercise, once a day for $1.5 \mathrm{~h}$. Campus regime included similar combination of exercises, but of higher intensity, twice per day, lasting 3 hours in total.

The research protocol started at $8 \mathrm{AM}$, after an overnight rest and fast, and before the breakfast. After filling out standard sports medicine questionnaire and passing the standard sports medicine examination, the blood samples were taken from the players.

The players (male or female, respectively) were randomly divided into two treatment groups. The supplemented group ( $n=8$ for male, $n=10$ for female) received $100 \mathrm{~mL}$ of chokeberry juice (Aronia anti-oxi donated by Nutrika d.o.o Belgrade, Serbia), in the morning during breakfast, for 4 weeks. The analytical analysis showed that $100 \mathrm{~mL}$ of the chokeberry juice contained $586.7 \pm 3.3 \mathrm{mg}$ of total phenolics expressed as galic acid equivalents (Kardum et al. 2014a), while the content of vitamin C was about $29 \mathrm{mg}$. In the same period, placebo group ( $n=7$ for male, $n=7$ for female) was given $100 \mathrm{~mL}$ of placebo, having the same content 
123 of vitamin $\mathrm{C}$ but no polyphenols. The placebo was identical to the chokeberry juice in terms 124 of taste and appearance. Therefore, the paricipants were not aware of the difference in the 125 juice consumed. Both investigators and the trainers were also blind to group assignment. 126 Compliance was monitored by the trainers.

127

128

\subsection{Anthropometric measurements}

Standing height was measured to the nearest $0.1 \mathrm{~cm}$ on a wall-mounted stadiometer with participants removing their shoes and socks (Perspective Enterprises, Kalamazoo, Mich., USA). Body weight to the nearest $0.1 \mathrm{~kg}$ and body composition were measured using Tanita body composition analyzer (TBF-300, Tanita Corp., Japan).

\subsection{Serum samples and biochemical determination}

Blood samples were taken at the beginning of the study and after four weeks chokeberry juice or placebo consumption during the preparatory training in campus. The samples were taken into sample tubes for serum and ethylenediaminetetraacetic acid (EDTA) tubes for plasma. Lipid status and glucose level were determined in sera of the athletes, on the same day the samples were collected, using the automated enzymatic methods (Roche Diagnostics kits, Mannheim, Germany), on Cobas e411 analyzer (Roche Diagnostics, Basel, Switzerland) according to the manufactures' instruction. Samples from the EDTA tubes were centrifuged at $1500 \mathrm{~g}$ for 5 minutes, and the obtained plasma stored at $-20^{\circ} \mathrm{C}$ until analysis.

The degree of lipid peroxidation in plasma was estimated by measuring of thiobarbituric acid reactive substances (TBARS) using 1\% TBA (Thiobarbituric Acid) in $0.05 \mathrm{NaOH}$, incubated with plasma at $100^{\circ} \mathrm{C}$ for $15 \mathrm{~min}$ and read at $530 \mathrm{~nm}$. Distilled water was used as a blank probe. TBA extract was obtained by mixing $0.8 \mathrm{ml}$ plasma and $0.4 \mathrm{ml}$ TCA (Trichloro Acetic 
147 Acid), then samples were put on ice for $10 \mathrm{~min}$, and centrifuged for $15 \mathrm{~min}$ at $6000 \mathrm{rpm}$. This 148 method was described previously (Okhava et al. 1979).

149

150

151

152

153

154

155

156

157

158

159

160

161

162

163

164

165

166

167

\subsection{Analysis of plasma phospholipids fatty acid composition}

Plasma lipids were extracted by the method of Folch et al. (1957) using a chloroformmethanol mixture $(2: 1 \mathrm{v} / \mathrm{v})$, as previously described (Petrovic et al. 2014). The 2,6-di-tertbutyl-4-methylphenol (BHT; $10 \mathrm{mg} / 100 \mathrm{ml}$ ) was added as an antioxidant. The phospholipids fraction was derived from the lipid extract by one-dimensional thin-layer chromatography (TLC) on Silica Gel GF plates (Merck, Darmstadt, Germany), using neutral lipid solvent system of hexane: diethyl ether: acetic acid (87:12:1). Methyl esters of phospholipids fatty acids were obtained by transmethylation, as already reported by Tepsic et al. (2009). Gas Chromatography (GC) using Shimadzu GC 2014 (Shimadzu Co, Tokyo, Japan) equipped with a flame ionization detector and Rtx 2330 fused silica gel capillary column, $(60 \mathrm{~m} \mathrm{x}$ $0.25 \mathrm{~mm} \times 0.2 \mu \mathrm{m}$ ) (Restek Co, Bellefonte, PA, USA) was applied to separate fatty acids methyl esters. Individual FA methyl esters in the samples were identified by comparing sample peak retention times with authentic standards (Sigma Chemical Co, St Louis, MO, USA) and/or (PUFA)-2 standard mixture (Restek Co, Bellefonte, PA, USA). Phospholipids FA profiles were expressed as the relative percentage areas of total FA.

The activities of enzymes involved in FA biosynthesis, desaturases and elongases, were estimated as the product-to-precursor ratios, as previously described (Petrovic et al. 2014). Estimate of $\Delta 5$-desaturase was computed using the $20: 4 n-6 / 20: 3 n-6$ ratio, while the $20: 3 n-6 / 18: 2 n-6$ ratio was used as a measure of $\Delta 6$-desaturase and elongase activities. The estimated $\Delta 9$-desaturase and elongase activities were derived from 18:1/18:0 and 18:0/16:0 ratios, respectively. 
172

173

174

175

176

177

178

179

180

181

182

183

184

185

186

187

188

189

190

191

192

193

194

195

196

\subsection{Statistical analysis}

The data are presented as mean values \pm standard deviation. Normal distribution was evaluated using Shapiro-Wilk test prior to statistical analysis. For non-normally distributed variables (18:3n-3, 22:4n-6 and MUFA), a logarithmic transformation was performed before comparison was made. Paired Student t-test was applied for the start vs. end of the study, and independent samples t-test for aronia vs. placebo group at the same time point for normally distributed variables. The statistical significance was defined as $p \leq 0.05$.

\section{Results}

3.1. Anthropometric and biochemical parameters of male and female handball players treated with chokeberry juice and placebo

As it can be seen from the Table 1, there were no differences in the anthropometric parameters both before and after the study in the same group, nor between chokeberry and placebo groups.

In both male and female athletes, there was no difference in serum glucose and total cholesterol concentration before and after the intervention with chokeberry juice or placebo (Table 2). However, triacylglycerol (TAG) concentration significantly decreased in male and increased in female handball players after the treatment with chokeberry juice or placebo $(p<$ $0.05)$

Lipid peroxidation was decreased in male players who consumed chokeberry juice, but not in the placebo controlled group nor in the female players.

\subsection{Fatty acid profile of plasma phospholipids in placebo and chokeberry juice-treated male} and female handball players

As presented in Table 3, consumption of chokeberry juice $(100 \mathrm{ml} /$ day $)$ in parallel with intense and regular physical training during 4 weeks, induced slight changes in FA profile of 
197 plasma phospholipids in male handball players. The significant decrease was observed only in 198 oleic acid (18:1 n-9) and $\alpha$-linolenic acid (18:3 n-3, ALA) after the treatment. However, 199 consumption of placebo with the same nutritional and training regimes led to significant 200 decreases of palmitoleic acid (16:1 n-7), vaccenic acid (18:1 n-7), ALA, eicosapentaenoic 201 acid (20:5 n-3, EPA) and docosatetraenoic acid (22:4 n-6) (Table 3).

Although the female players were subjected to the same intervention, the obtained results are different. While chokeberry juice induced no changes after 4 weeks, n-6 PUFA and consequently total PUFA were reduced in the placebo controlled group (Table 4). Interestingly, estimated desaturase and elongase activities were not amended in both male and female handball players after the treatment with chokeberry juice or placebo (Table 5).

207

\section{Discussion}

In elite athletes chronic intensive training leads to oxidative stress and metabolic changes of lipid and FA profiles. Since lipids, especially PUFA, are the most susceptible targets for free radicals induced damages, polyphenol rich chockeberry juice could protect membrane phospholipids from oxidative damage. However, in this study no significant increase in PUFA was found in male and female handball players after 4 weeks of chokeberry juice consumption.

All study participants had biochemical parameters within the normal range, including serum TAG levels. After 4 weeks of preparatory training in the male players serum TAG concentration was further reduced. In accordance, reduced plasma TAG concentration was reported in sportsmen participating in other sports, as in long distance runners, cyclists, soccer players and swimmers (Kelley and Kelley 2009; Lira et al. 2010; Rahnama et al. 2009; Saldana et al. 1995). This can be explained by the increased metabolism and utilization of

221 TAG to meet higher energy demands in endurance exercise (Kelley and Kelley 2009). On the 
222 other hand, in female players TAG concentration increased in both placebo and chokeberry

223 groups. Although previous studies reported lipid-lowering effect of chokeberry extracts

224 (Sikora et al. 2014; Skoczynska et al. 2007) these studies have been conducted in subjects 225 with hyperlipidemia and/or metabolic syndrome. Elevated levels of TAG in female players 226 may be attributed to enhanced lipid mobilization from adipose tissue during intensified 227 trainings in the campus. In addition, the observed differences between males and females 228 could be explained by sex differences in exercise metabolism (Tarnopolsky 2008; Wu and 229 O'Sullivan 2011). Women use fat as a main energy source for endurance exercise, mostly due 230 to the higher fat mass and greater adipocyte lipolysis. Compared with men, they have less 231 muscle glycogen utilization and higher plasma free fatty acid during endurance exercise 232 (Hamadeh et al. 2005; Roepstorff et al. 2002). These sex differences are due to differences in estrogen concentration and/or activity (Mittendorfer et al. 2002). This could explain transient elevation in TAG concentration in female players after intensive trainings in campus.

Plasma TBARS levels are the most often used marker of lipid peroxidation and oxidative damage in general. Thus we determined TBARS at the beginning and at the end of the study. Male players who consumed chokeberry juice showed decreased levels of TBARS after the study period, unlikely those who consumed placebo. These results are in accordance with previous studies (Pilaczynska-Szczesniak et al. 2005). Since both the juice and placebo contained vitamin C (around $29 \mathrm{mg}$ ), which is well known antioxidant, the observed differences occurred due to polyphenols and their strong antioxidative capacity. However, in female athletes we found no changes in TBARS level in both groups, although Kardum et al. (2014b) have recently reported reduced TBARS in healthy sedentary women after consumption of chokeberry juice. This suggests that the effect of intensive training, which 
TBARS in females. Previous studies have also shown that lipid peroxidation during exercise is higher in women than in men due to effect of estrogen (Devries et al. 2007).

The fatty acid composition of plasma phospholipids followed in this study, showed some treatment- and sex-dependent alternations. Total SFA, MUFA n-3 and n-6 PUFA (except n-6 PFA in the female placebo group) remained unchanged after the training cycle in both males and females and regardless of the juice consumption. Similarly, no differences were found in the activities of enzymes responsible for FA metabolism. Moderate differences were found in specific FA. In placebo-treated male handball players significant reduction of MUFA: palmitoleic and vaccenic acid, and anti-inflammatory PUFA: ALA (n-3), EPA (n-3) and docosatetraenoic acid (n-6) was found. These effects were mostly attenuated by the intake of chokeberry juice and these sportsmen had lower levels of ALA and oleic acid after the treatment. At the same time, chokeberry juice intake produced no significant alternations in proportion of individual fatty acids in female athletes, while in placebo group a decrease of $n$ 6 and consequently total PUFA was observed.

Interestingly, all FA which proportion was reduced in male handball players at the end of the study have beneficial effects on health. Palmitoleic acid plays roles in different physiological processes from cell growth and proliferation, to de novo fat synthesis and storage (De Fabiani 2011). It exerts anti-apoptotic activity, increases insulin sensitivity by suppressing inflammation, and inhibits the destruction of insulin-secreting pancreatic $\beta$-cells (Chajes et al. 2011). The pro-lipogenic activity of palmitoleic acid in the adipose tissue protects other tissues and organs from TAG accumulation and lipotoxicity (Cao et al. 2008; Scherer et al. 2011). The other two MUFAs, vaccenic acid (18:1n-7, VV) and oleic acid (18:1, n-9), declined in sportsmen treated with placebo and chokeberry, respectively. These FA are both associated with improvement of cell membrane fluidity and with the lower risk of coronary heart disease (Djousse et al. 2012; Haug et al. 2007). Furthermore, both groups of 
271 male players had lower level of ALA after the study. ALA is an essential FA, precursor of

272 long chain n-3 PUFAs and its decrease could be a consequence of intensive training or

273 different intake in the campus. Together with EPA (which level was reduced in the placebo

274 group but not in the chokeberry treated sportsmen), ALA has important role in prevention of

275 different physiological disorders, including hyperlipidemia, inflammation, loss of bone

276 mineral and coronary heart disease (Vucic and Ristic-Medic 2012). The decrease of n-6

277 docosatetraenoic acid can affect prostaglandin and thromboxane synthesis, having an impact

278 on aortic endothelial cells and platelet function (Campbell et al. 1985). However, the results

279 of the same study in female players are different. Chokeberry consumption induced no effect

280 in serum FA profiles, while in placebo controlled group a decrease of n-6 and total PUFA,

281 which could be mainly attributed to a decrease of linoleic acid, has been found. n-6 PUFA are

282 generally known to give rise to pro-inflammatory eicosanoids (prostaglandin $\mathrm{E}_{2}$ and

283 leukotriene $\mathrm{B}_{4}$ ), potent lipid signaling molecules which are the mediators of various

284 pathophysiological processes as asthma, obesity, rheumatoid arthritis, atherosclerosis, and

285 cardiovascular disease (Burtscher and Gnaiger 2013; Grimble 2012). A possible reason for

286 decreased PUFA in female athletes, could be lipid peroxidation induced by the oxidative

287 stress (Dunford and Doyle 2015). The results previously published from our laboratory

288 indicated a significant increase of the antioxidant enzymes activity (superoxide dismutase and

289 glutathione peroxidase) and the decrease of pro-oxidant-antioxidant balance and lipid

290 peroxidation in apparently healthy subject after the long-term (12 weeks) chokeberry juice

291 consumption (Kardum et al. 2014a, b). Nevertheless, we did not find these effects in active

292 handball players. When compared the FA profiles of the chokeberry consuming groups at the

293 beginning and at the end of the study, we found slight differences in male and no differences

294 in female players. When compared chokeberry vs placebo group, there was no significant

295 changes in any FA. In line with this is the result on the estimated desaturase and elongase 
activities which remained unaltered in all study groups, suggesting that the observed changes in FA profiles are not due to amended metabolism of FA.

The weakness of this study is relatively small number of study participants per study group, which is conditioned by the limited number of players chosen for the National team and/or elite clubs. It is particularly important since most changes in FA profiles are found in those FA that are presented in small amounts. Another limitation is the lack of the data on hormonal status of the study participants (progesterone/estradiol ratio) which may affect redox status (Devries et al. 2007). Although we can assume that female players were not in the same phase of menstrual cycle, we did not find striking differences in the studied parameters among them, which could be attributed to the differences in hormonal status. Furthermore, longer treatment with a greater amount of chokeberry juice might lead to desirable changes in FA profiles. According to our results we can conclude that handball players during the period of preparatory training should have nutritional intervention and/or supplementation primarily with n-3 PUFA, while chokeberry juice had weak beneficial effects in these athletes.

\section{Acknowledgements}

This study was supported by Grants No. III 41030 and 175043 from the Ministry of Education, Science and Technological Development of the Republic of Serbia. We are also grateful to Nutrika d.o.o. Belgrade, Serbia for supplying chokeberry juice.

\section{REFERENCES}

Arsic, A., Vucic, V., Tepsic, J., Mazic, S., Djelic, M., and Glibetic, M. 2012. Altered plasma and erythrocyte phospholipid fatty acid profile in elite female water polo and football players. Appl. Physiol. Nutr. Metab. 37(1): 40-47. 
Arsic, A., Vucic, V., Glibetic, M., Popovic, T., Debeljak-Martacic, J., Cubrilo, D., et al. 2016. Redox balance in elite female athletes: differences based on sport types. J. Sport Med. Phys. Fit. 56(1-2): 1-8.

Bermudez-Soto, M.J., and Tomas-Barberan, F.A. 2004. Evaluation of commercial red fruit juice concentrates as ingredients for antioxidant functional juices. Eur. Food Res. Technol. 219: $133-141$.

Broncel, M., Kozirog, M., Duchnowicz, P., Koter-Michalak, M., Sikora, J., and ChojnowskaJezierska, J. 2010. Aronia melanocarpa extract reduces blood pressure, serum endothelin, lipid, and oxidative stress marker levels in patients with metabolic syndrome. Med. Sci. Monit. 16(1): CR 28-34.

Burtscher, M., and Gnaiger E. 2013. Physical activity and cardiovascular diseases epidemiology and primary preventive and therapeutic targets. In Interdisciplinary concepts in cardiovascular health. Edited by I. Wakabayashi and K. Groschner. Springer-Verlag, Wien, Austria. pp. 127-144.

Campbell, W.B., Falck, J.R., Okita, J.R., Johnson, A.R., and Callahan, K.S. 1985. Synthesis of dihomoprostaglandins from adrenic acid (7,10,13,16-docosatetraenoic acid) by human endothelial cells. Biochim. Biophys. Acta, 837(1): 67-76.

Cao, H., Gerhold, K., Mayers, J.R., Wiest, M.M., Watkins, S.M., and Hotamisligil, G.S. 2008. Identification of a lipokine, a lipid hormone linking adipose tissue to systemic metabolism. Cell, 134: 933-944.

Chajes, V., Joulin, V., and Clavel-Chapelon, F. 2011. The fatty acid desaturation index of blood lipids, as a biomarker of hepatic stearoyl-CoA desaturase expression, is a predictive factor of breast cancer risk. Curr. Opin. Lipidol. 22(1): 6-10.

De Fabiani, E. 2011. The true story of palmitoleic acid: Between myth and reality. Eur. J. Lipid. Sci. Technol. 113(7): 809-811. 
Devries, M.C., Lowther, S.A., Glover, A.W., Hamadeh, M.J., and Tarnopolsky, M.A. 2007. IMCL area density, but not IMCL utilization, is higher in women during moderate-intensity endurance exercise, compared with men. Am. J. Physiol. 293(6): R2336-2342.

Djousse, L., Matthan, N.R., Lichtenstein, A.H., and Gaziano, J.M. 2012. Red Blood Cell Membrane Concentration of Cis-Palmitoleic and Cis-Vaccenic Acids and Risk of Coronary Heart Disease. Am. J. Cardiol. 110(4): 539-544.

Dunford, M., and Doyle, J. 2015. Nutrition for sport und exercise (3rd ed.). Stamford (USA): Cengage Learning.

Grimble, R.F. 2012. Cytokines and nutrition. In Artificial nutrition and support in clinical practice. Edited by J. Payne-James, G.K. Grimble, and D.B.A. Silk. Cambridge University Press, Cambridge, United Kingdom. pp. 93-106.

Hamadeh, M.J., Devries, M.C., and Tarnopolsky, M.A. 2005. Estrogen supplementation reduces whole body leucine and carbohydrate oxidation and increases lipid oxidation in men during endurance exercise. J. Clin. Endocrinol. Metab. 90(6): 3592-3599.

Haug, A., Hostmark, A.T., and Harstad, O.M. 2007. Bovine milk of human nutrition - a review. Lipids Health Dis. 6(1): 25.

Kardum, N., Petrovic-Oggiano, G., Takic, M., Glibetic, N., Zec, M., Debeljak-Martacic, J., et al. 2014a. Effects of glucomannan-enriched, aronia juice-based supplement on cellular antioxidant enzymes and membrane lipid status in subjects with abdominal obesity. Sci. World J. 2014: 869250.

Kardum, N., Takic, M., Šavikin, K., Zec, M., Zdunic, G., Spasic, S., et al. 2014b. Effects of polyphenol-rich chokeberry juice on cellular antioxidant enzymes and membrane lipid stat us in healthy women. J. Funct. Foods, 9(1): 89-97.

Kelley, G.A., and Kelley, K.S. 2009. Impact of progressive resistance training on lipids and lipoproteins in adults: a meta-analysis of randomized controlled trials. Prev. Med. 48(1): 9-19. 
Kulling, S.E., and Rawel, H.M. 2008. Chokeberry (Aronia melanocarpa)-A Review on the Characteristic Components and Potential Health Effects. Planta Med. 74(13): 1625-1634.

Lafay, S., Jan, C., Nardon, K., Lemaire, B., Ibarra, A., Roller, M., et al. 2009. Grape extract improves antioxidant status and physical performance in elite male athletes. J. Sport Sci. Med. 8(3): 468-480.

Lira, F.S., Rosa, J.C., Lima-Silva, A.E., Souza, H.A., Caperuto, E.C., Seelaender, M.C., et al. 2010. Sedentary subjects have higher PAI-1 and lipoproteins levels than highly trained athletes. Diabetol. Metab. Syndr. 2:7. doi: 10.1186/1758-5996-2-7.

Margaritis I., Palazzetti S., Rousseau A., Richard M., and Favier A. 2003. Antioxidant supplementation and tapering exercise improve exercise-induced antioxidant response. J. Am. Coll. Nutr. 22(2): 147-156.

Marin, D.P., dos Santos, M., de Cassia, R., Bolin, A.P., Guerra, B.A., Hatanaka, E., et al. 2011. Cytokines and oxidative stress status following a handball game in elite male players. Oxid. Med. Cell Longev. 2011:804873. doi: 10.1155/2011/804873

Mittendorfer, B., Horowitz, J.F., and Klein, S. 2002. Effect of gender on lipid kinetics during endurance exercise of moderate intensity in untrained subjects. Am. J. Physiol. 283: E58 E65.

Morillas-Ruiz, J., Zafrilla, P., Almar, M., Cuevas, M. J., Lopez, F.J., Abellan, P., et al. 2005. The effects of an antioxidant-supplemented beverage on exercise-induced oxidative stress: results from a placebo-controlled double-blind study in cyclists. Eur. J. Appl. Physiol. 95(5-6):543-549.

Morillas-Ruiz, J., Villegas Garcia, J., Lopez F., Vidal-Guevara, M., and Zafrill,a P. 2006. Effects of polyphenolic antioxidants on exercise-induced oxidative stress. Clin. Nutr. 25(3): 444-453. 
394 Mougios, V., Kotzamanidis, C., Koutsari, C., and Atsopardis, S. 1995. Exercise-induced 395 changes in the concentration of individual fatty acids and triacylglycerols of human 396 plasma. Metabolism, 44(5): 681-688.

397 Ohkawa, H., Ohishi, N., and Yagi, K. 1979. Assay for lipid peroxides in animal tissues by 398 thiobarbituric acid reaction. Anal. Biochem. 95(2): 351-358.

399 Petrović, S., Takić, M., Arsić, A., Vučić, V., Drakulić, D., Milošević, M., et al. 2014. Effect 400 of sex hormones on plasma phospholipid fatty acid composition in intact rats and rats with 401 bilaterally occluded carotid arteries. Physiol. Res. 63(3): 331-339.

402 Pilaczynska-Szczesniak, L., Skarpanska-Steinborn, A., Deskur, E., Basta, P., and 403 Horoszkiewicz-Hassan, M. 2005. The influence of chokeberry juice supplementation on the 404 reduction of oxidative stress resulting from an incremental rowing ergometer exercise. Int. J. 405 Sport Nutr. Exerc. Metab. 15(1): 48-58.

406 Rahnama, N., Younesian, A., Mohammadion, M., and Bambaeichi, E. 2009. A 90 minute 407 soccer match decreases triglyceride and low density lipoprotein but not high-density 408 lipoprotein and cholesterol levels. J. Res. Med. Sci. 14(6): 335-341.

409 Roepstorff, C., Steffensen, C.H., Madsen, M., Stallknect, B., Kanstrup, .I, Richter, E.A., et al. 410 2002. Gender differences in substrate utilization during submaximal exercise in endurance411 trained subjects. Am. J. Physiol. 282: E435-E447.

412 Scherer, T., O'Hare, J., Diggs-Andrews, K., Schweiger, M., Cheng, B., Lindtner, C., et al. 413 2011. Brain insulin controls adipose tissue lipolysis and lipogenesis. Cell Metab. 13(2): 183414194.

415 Sikora, J., Broncel, M., and Mikiciuk-Olasik, E. 2014. Aronia melanocarpa Elliot Reduces 416 the Activity of Angiotensin I-Converting Enzyme-In Vitro and Ex Vivo Studies. Oxid. Med. 417 Cell Longev. 2014: 739721. doi: 10.1155/2014/739721. 
418 Skoczynska, A., Jedrychowska, I., Poreba, R., Affelska-Jercha, A., Turczyn, B., Wojakowska 419 A., et al. 2007. Influence of chokeberry juice on arterial blood pressure and lipid parameters 420 in men with mild hypercholesterolemia. Pharmacol. Rep. 59(1): 177-182.

421 Solans, R., Motta, C., Solá, R., La Ville, A.E., Lima, J., Simeón, P., et al. 2000. Abnormalities 422 of erythrocyte membrane fluidity, lipid composition, and lipid peroxidation in systemic 423 sclerosis: evidence of free radical-mediated injury. Arthritis Rheum. 43(4): 894-900.

424 Szajdek, A., and Borowska, E.J. 2008. Bioactive compounds and health-promoting properties 425 of berry fruits: A review. Plant Food Hum. Nutr. 63(4): 147-156.

426 Tarnopolsky, M.A. 2008. Sex differences in exercise metabolism and the role of 17-beta 427 estradiol. Med. Sci. Sport Exer. 40(4): 648-654.

428 Tepsic, J., Vucic, V., Arsic, A., Blazencic-Mladenovic, V., Mazic, S., and Glibetic, M. 2009. 429 Plasma and erythrocyte phospholipid fatty acid profile in professional basketball and football 430 players. Eur. J. Appl. Physiol. 107(3): 359-365.

431 Tepsic, J., Vucic, V., Arsic, A., Mazic, S., Djelic, M., and Glibetic, M. 2013.Unfavourable 432 plasma and erythrocyte phospholipid fatty acid profile in elite amateur boxers. Eur. J. Sport 433 Sci. 13(4): 414-421.

434 Vucic, V., and Ristic-Medic, D. 2012. Eicosapentaenoic acid: The role in malignant diseases. 435 In Eicosapentaenoic acid: Sources, health effects and role in disease prevention. Edited by 436 T.G. Bradley, and F.P, Vargas. Nova Science Publishers, New York, USA. pp. 99-116.

437 Wu, B. N., and O'Sullivan, A. J. 2011. Sex differences in energy metabolism need to be 438 considered with lifestyle modifications in humans. J. Nutr. Metab. 2011: 391809. doi: $43910.1155 / 2011 / 391809 .$.

440 Zheng, W., and Wang, S.Y. 2003. Oxygen radical absorbing capacity of phenolics 441 inblueberries, cranberries, chokeberries, and lingonberries. J. Agric. Food Chem. 51(2):502442509. 
443 Table 1. The anthropometric characteristics of male and female handball players and 444 responsiveness to chokeberry juice and placebo

\begin{tabular}{|c|c|c|c|c|}
\hline & $\begin{array}{l}\text { Chokeberry } \\
\text { juice }(n=8)\end{array}$ & & Placebo $(n=7)$ & \\
\hline Men & $\begin{array}{l}\text { Before } \\
\text { treatment }\end{array}$ & $\begin{array}{l}\text { After } \\
\text { treatment }\end{array}$ & Before treatment & After treatment \\
\hline Age & $18.57 \pm 0.53$ & $18.80 \pm 0.58$ & $18.38 \pm 1.41$ & $18.57 \pm 0.53$ \\
\hline Height $(\mathrm{cm})$ & $192.86 \pm 6.91$ & $192.86 \pm 6.91$ & $192.63 \pm 6.23$ & $195.33 \pm 4.37$ \\
\hline Weight (kg) & $89.81 \pm 12.02$ & $90.27 \pm 12.32$ & $91.40 \pm 17.95$ & $97.13 \pm 18.77$ \\
\hline WHR & $0.78 \pm 0.04$ & $0.76 \pm 0.03$ & $0.84 \pm 0.09$ & $0.85 \pm 0.10$ \\
\hline BMI $\left(\mathrm{kg} / \mathrm{m}^{2}\right)$ & $24.10 \pm 2.63$ & $23.63 \pm 2.72$ & $24.61 \pm 4.78$ & $25.62 \pm 5.67$ \\
\hline $\begin{array}{l}\text { Basal metabolism } \\
\text { (kcal) }\end{array}$ & $2163 \pm 232$ & $2212 \pm 230$ & $2088 \pm 186$ & $2188 \pm 160$ \\
\hline Body fat $(\%)$ & $7.43 \pm 2.47$ & $5.34 \pm 2.57$ & $8.40 \pm 2.78$ & $6.63 \pm 3.21$ \\
\hline Free fat mass $(\mathrm{kg})$ & $6.82 \pm 4.54$ & $5.04 \pm 3.66$ & $7.75 \pm 3.45$ & $6.35 \pm 3.15$ \\
\hline \multirow[t]{2}{*}{$\begin{array}{l}\text { Total body water } \\
(\mathrm{kg})\end{array}$} & $60.93 \pm 7.96$ & $62.56 \pm 7.91$ & $58.40 \pm 6.06$ & $61.73 \pm 5.34$ \\
\hline & $\begin{array}{l}\text { Chokeberry } \\
\text { juice }(n=10)\end{array}$ & & $\begin{array}{l}\text { Placebo } \\
(n=7)\end{array}$ & \\
\hline Women & Before & After & Before treatment & After treatment \\
\hline
\end{tabular}




treatment treatment

\begin{tabular}{|c|c|c|c|c|}
\hline Age & $16.8 \pm 0.6$ & $17.1 \pm 0.7$ & $17.3 \pm 1.3$ & $17.5 \pm 1.7$ \\
\hline Height (cm) & $172.8 \pm 6.5$ & $172.2 \pm 7.3$ & $168.3 \pm 8.8$ & $169.4 \pm 8.7$ \\
\hline Weight (kg) & $65.8 \pm 7.8$ & $66.3 \pm 7.7$ & $64.0 \pm 11.4$ & $66.2 \pm 11.8$ \\
\hline WHR & $0.84 \pm 0.02$ & $0.85 \pm 0.04$ & $0.84 \pm 0.05$ & $0.85 \pm 0.05$ \\
\hline BMI $\left(\mathrm{kg} / \mathrm{m}^{2}\right)$ & $21.5 \pm 5.4$ & $22.3 \pm 2.5$ & $22.8 \pm 2.0$ & $22.9 \pm 2.0$ \\
\hline $\begin{array}{l}\text { Basal metabolism } \\
\text { (kcal) }\end{array}$ & $1489 \pm 138$ & $1498 \pm 150$ & $1394 \pm 175$ & $1426 \pm 167$ \\
\hline Body fat (\%) & $21.06 \pm 5.41$ & $21.02 \pm 6.86$ & $25.80 \pm 1.70$ & $25.96 \pm 3.45$ \\
\hline Free fat mass $(\mathrm{kg})$ & $14.33 \pm 3.93$ & $14.81 \pm 6.96$ & $15.18 \pm 3.95$ & $14.80 \pm 4.86$ \\
\hline $\begin{array}{l}\text { Total body water } \\
(\mathrm{kg})\end{array}$ & $38.05 \pm 4.69$ & $38.24 \pm 5.11$ & $34.75 \pm 5.95$ & $35.78 \pm 5.69$ \\
\hline
\end{tabular}

449

450

451

452 
453 Table 2. Serum lipid status and glucose of handball players (male - M and female $-F$ ) before 454 and after the treatment with chokeberry juice or placebo.

455

\begin{tabular}{lllll}
\hline \multicolumn{2}{l}{ Chokeberry juice } & \multicolumn{2}{l}{ Placebo } & \\
\hline Parameter $(\mathrm{mmol} / \mathrm{L})$ & Before & After & Before & After treatment \\
& treatment & treatment & treatment & \\
\hline Glucose & & & & \\
M & $4.47 \pm 0.29$ & $4.27 \pm 0.30$ & $4.84 \pm 0.30$ & $4.33 \pm 0.47$ \\
F & $4.42 \pm 0.26$ & $4.32 \pm 0.41$ & $4.61 \pm 0.29$ & $4.41 \pm 0.41$
\end{tabular}

Triacylglycerol

$\begin{array}{lllll}\mathrm{M} & 0.87 \pm 0.19 & 0.64 \pm 0.22^{*} & 0.96 \pm 0.28 & 0.64 \pm 0.13^{*} \\ \mathrm{~F} & 0.47 \pm 0.12 & 0.67 \pm 0.17 * & 0.57 \pm 0.13 & 0.73 \pm 0.12^{*}\end{array}$

Total cholesterol

$\begin{array}{lllll}\mathrm{M} & 4.06 \pm 0.61 & 3.72 \pm 0.46 & 4.08 \pm 0.45 & 3.90 \pm 0.51 \\ \mathrm{~F} & 3.94 \pm 0.93 & 4.38 \pm 0.46 & 3.93 \pm 0.47 & 4.14 \pm 0.45\end{array}$

TBARS

$\begin{array}{lllll}\mathrm{M} & 0.63 \pm 0.21 & 0.33 \pm 0.25^{*} & 0.58 \pm 0.38 & 0.56 \pm 0.24 \\ \mathrm{~F} & 6.68 \pm 0.35 & 7.71 \pm 1.05 & 6.58 \pm 0.22 & 7.02 \pm 1.04\end{array}$

456

457 Data are presented as a mean \pm SD. ${ }^{*} p<0.05$ compared to baseline (before treatment).

458

459

460

461 
462 Table 3. Plasma phospholipids fatty acid composition in male handball players before and 463 after the treatment with chokeberry juice and placebo

\begin{tabular}{|c|c|c|c|c|}
\hline & Chokeberry juic & & Placebo & \\
\hline $\begin{array}{l}\text { Fatty acid } \\
(\%)\end{array}$ & Before treatment & After treatment & Before treatment & After treatment \\
\hline $16: 0$ & $26.59 \pm 2.06$ & $28.07 \pm 1.60$ & $26.20 \pm 2.51$ & $27.53 \pm 1.76$ \\
\hline 18:0 & $14.79 \pm 0.82$ & $14.46 \pm 1.27$ & $15.68 \pm 1.46$ & $15.12 \pm 1.02$ \\
\hline SFA & $41.38 \pm 1.68$ & $42.53 \pm 1.82$ & $41.88 \pm 1.22$ & $42.65 \pm 1.39$ \\
\hline $16: 1 n-7$ & $0.54 \pm 0.29$ & $0.38 \pm 0.14$ & $0.60 \pm 0.17$ & $0.38 \pm 0.12 *$ \\
\hline $18: 1 n-9$ & $9.31 \pm 1.34$ & $8.23 \pm 0.71 *$ & $8.49 \pm 0.97$ & $8.10 \pm 0.70$ \\
\hline $18: 1 n-7$ & $1.60 \pm 0.24$ & $1.62 \pm 0.15$ & $1.53 \pm 0.22$ & $1.31 \pm 0.21 *$ \\
\hline MUFA & $11.44 \pm 1.72$ & $10.23 \pm 0.69$ & $10.62 \pm 0.97$ & $9.79 \pm 0.69$ \\
\hline $18: 2 n-6$ & $26.96 \pm 2.66$ & $27.20 \pm 2.41$ & $26.03 \pm 2.79$ & $27.46 \pm 3.33$ \\
\hline $20: 3 n-6$ & $3.40 \pm 0.76$ & $3.48 \pm 0.53$ & $3.22 \pm 0.41$ & $3.22 \pm 1.03$ \\
\hline $20: 4 n-6$ & $12.44 \pm 2.07$ & $12.19 \pm 1.41$ & $13.79 \pm 2.73$ & $12.85 \pm 2.35$ \\
\hline $22: 4 n-6$ & $0.61 \pm 0.08$ & $0.57 \pm 0.10$ & $0.78 \pm 0.21$ & $0.62 \pm 0.12 *$ \\
\hline n-6 PUFA & $43.42 \pm 2.67$ & $43.43 \pm 2.40$ & $43.82 \pm 1.72$ & $44.15 \pm 1.41$ \\
\hline $18: 3 n-3$ & $0.26 \pm 0.15$ & $0.13 \pm 0.02 *$ & $0.41 \pm 0.17$ & $0.13 \pm 0.05 * * *$ \\
\hline $20: 5 n-3$ & $0.38 \pm 0.07$ & $0.30 \pm 0.10$ & $0.38 \pm 0.09$ & $0.24 \pm 0.10 * *$ \\
\hline $22: 5 n-3$ & $0.70 \pm 0.15$ & $0.75 \pm 0.09$ & $0.62 \pm 0.16$ & $0.64 \pm 0.10$ \\
\hline
\end{tabular}


$\begin{array}{lllll}22: 6 n-3 & 2.41 \pm 0.77 & 2.56 \pm 0.56 & 2.31 \pm 0.17 & 2.41 \pm 0.39\end{array}$

$\begin{array}{lllll}\text { n-3 PUFA } & 3.75 \pm 0.96 & 3.72 \pm 0.66 & 3.66 \pm 0.41 & 3.41 \pm 0.36\end{array}$

\begin{tabular}{lllll}
\hline $\mathbf{n}-6 / \mathbf{n}-3$ & $47.18 \pm 3.36$ & $47.16 \pm 2.31$ & $47.49 \pm 1.60$ & $47.56 \pm 1.44$
\end{tabular}

$\begin{array}{lllll}\text { ratio } & 12.10 \pm 2.47 & 11.97 \pm 2.15 & 12.11 \pm 1.61 & 13.06 \pm 1.36\end{array}$

PUFA/SFA

$\begin{array}{lllll}\text { PUFA/SFA } & 1.13 \pm 0.07 & 1.12 \pm 0.07 & 1.14 \pm 0.13 & 1.11 \pm 0.10 \\ \text { ratio } & & & & \end{array}$

464

465

466

467

468

469

470

471

472

473

474

475

477

478

479

480

480

Data are presented as a mean \pm SD.

SFA, saturated fatty acids; MUFA, monounsaturated fatty acids; PUFA, polyunsaturated fatty acids. ${ }^{*} p<0.05,{ }^{*} p<0.01, * * * p<0.001$ compared to baseline (before treatment).

(1)

(1)

(1)

.

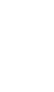

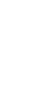

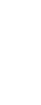

\section{6}

ratio 
481 Table 4. Serum phospholipids fatty acid composition in female handball players before and 482 after the treatment with chokeberry juice and placebo

\begin{tabular}{|c|c|c|c|c|}
\hline & Chokeberry juic & & Placebo & \\
\hline $\begin{array}{l}\text { Fatty acid } \\
(\%)\end{array}$ & Before treatment & After treatment & Before treatment & After treatment \\
\hline $16: 0$ & $28.07 \pm 1.32$ & $28.44 \pm 1.94$ & $27.90 \pm 1.89$ & $28.08 \pm 0.64$ \\
\hline $18: 0$ & $13.93 \pm 1.17$ & $14.47 \pm 1.52$ & $14.76 \pm 0.94$ & $15.47 \pm 0.80$ \\
\hline SFA & $42.00 \pm 1.23$ & $42.91 \pm 0.96$ & $42.67 \pm 1.35$ & $43.55 \pm 0.73$ \\
\hline $16: 1 n-7$ & $0.46 \pm 0.06$ & $0.42 \pm 0.12$ & $0.49 \pm 0.12$ & $0.56 \pm 0.13$ \\
\hline $18: 1 n-9$ & $8.52 \pm 0.91$ & $8.85 \pm 0.85$ & $7.81 \pm 0.49$ & $8.55 \pm 0.78$ \\
\hline $18: 1 n-7$ & $1.45 \pm 0.17$ & $1.40 \pm 0.23$ & $1.34 \pm 0.18$ & $1.39 \pm 0.19$ \\
\hline MUFA & $10.42 \pm 0.91$ & $10.67 \pm 0.99$ & $9.65 \pm 0.49$ & $10.50 \pm 0.71$ \\
\hline $18: 2 n-6$ & $29.51 \pm 2.66$ & $29.46 \pm 2.35$ & $29.85 \pm 1.64$ & $27.11 \pm 1.78$ \\
\hline $20: 3 n-6$ & $2.74 \pm 0.75$ & $2.71 \pm 0.69$ & $2.69 \pm 0.77$ & $3.34 \pm 0.62$ \\
\hline $20: 4 n-6$ & $11.15 \pm 1.63$ & $10.56 \pm 1.63$ & $11.10 \pm 1.47$ & $11.32 \pm 1.95$ \\
\hline $22: 4 n-6$ & $0.51 \pm 0.06$ & $0.46 \pm 0.12$ & $0.50 \pm 0.19$ & $0.61 \pm 0.11$ \\
\hline n-6 PUFA & $43.92 \pm 1.57$ & $43.20 \pm 1.12$ & $44.14 \pm 1.03$ & $42.37 \pm 0.57^{*}$ \\
\hline $18: 3 n-3$ & $0.26 \pm 0.17$ & $0.19 \pm 0.12$ & $0.17 \pm 0.15$ & $0.21 \pm 0.16$ \\
\hline $20: 5 n-3$ & $0.25 \pm 0.09$ & $0.23 \pm 0.12$ & $0.19 \pm 0.12$ & $0.24 \pm 0.08$ \\
\hline $22: 5 n-3$ & $0.52 \pm 0.11$ & $0.50 \pm 0.13$ & $0.54 \pm 0.22$ & $0.53 \pm 0.15$ \\
\hline
\end{tabular}


$\begin{array}{lllll}22: 6 n-3 & 2.63 \pm 0.49 & 2.29 \pm 0.63 & 2.64 \pm 0.75 & 2.59 \pm 0.39\end{array}$

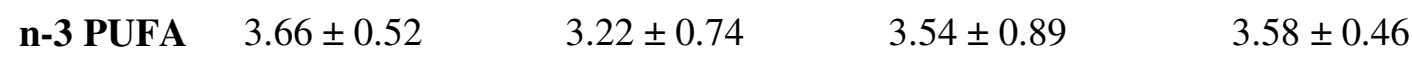

\begin{tabular}{lllll}
\hline PUFA & $47.58 \pm 1.51$ & $46.42 \pm 0.92$ & $47.69 \pm 1.10$ & $45.95 \pm 0.85^{*}$
\end{tabular}

n-6/n-3

ratio

$12.25 \pm 2.02 \quad 14.08 \pm 3.14 \quad 13.32 \pm 4.15 \quad 12.05 \pm 1.89$

\section{PUFA/SFA}
$1.13 \pm 0.07$
$1.08 \pm 0.04$
$1.12 \pm 0.06$
$1.06 \pm 0.03$

ratio

483

484

485

486

487

488

489

490

491

492

493

494

495

496

497

498

499

Data are presented as a mean \pm SD.

SFA, saturated fatty acids; MUFA, monounsaturated fatty acids; PUFA, polyunsaturated fatty acids. ${ }^{*} p<0.05$ compared to baseline (before treatment).

(

(1)

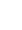

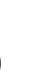

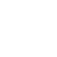

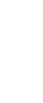


501 Table 5. The estimated plasma desaturase and elongase activities in male (M) and female (F)

502 handball players before and after the treatment with chokeberry juice and placebo

\begin{tabular}{|c|c|c|c|c|}
\hline \multirow[b]{2}{*}{ Desaturase and } & \multicolumn{2}{|c|}{ Chokeberry juice } & \multicolumn{2}{|l|}{ Placebo } \\
\hline & Before & After & Before & After \\
\hline elongase & treatment & treatment & treatment & treatment \\
\hline \multicolumn{5}{|c|}{$20: 4 n-6 / 20: 3 n-6(\Delta 5)$} \\
\hline M & $3.82 \pm 1.06$ & $3.56 \pm 0.55$ & $4.35 \pm 0.97$ & $4.38 \pm 1.58$ \\
\hline $\mathrm{F}$ & $4.34 \pm 1.24$ & $4.17 \pm 1.44$ & $4.39 \pm 1.37$ & $3.50 \pm 0.91$ \\
\hline \multicolumn{5}{|c|}{$20: 3 n-6 / 18: 2 n-6(\Delta 6)$} \\
\hline M & $0.13 \pm 0.04$ & $0.13 \pm 0.02$ & $0.13 \pm 0.02$ & $0.12 \pm 0.05$ \\
\hline $\mathrm{F}$ & $0.09 \pm 0.03$ & $0.09 \pm 0.03$ & $0.09 \pm 0.03$ & $0.12 \pm 0.03$ \\
\hline \multicolumn{5}{|l|}{$18: 1 / 18: 0(\Delta 9)$} \\
\hline $\mathrm{M}$ & $0.63 \pm 0.11$ & $0.57 \pm 0.07$ & $0.55 \pm 0.09$ & $0.54 \pm 0.05$ \\
\hline $\mathrm{F}$ & $0.62 \pm 0.11$ & $0.62 \pm 0.10$ & $0.53 \pm 0.05$ & $0.55 \pm 0.06$ \\
\hline \multicolumn{5}{|c|}{ 18:0/16:0 (elongase) } \\
\hline M & $0.56 \pm 0.06$ & $0.52 \pm 0.06$ & $0.61 \pm 0.12$ & $0.55 \pm 0.06$ \\
\hline $\mathrm{F}$ & $0.50 \pm 0.06$ & $0.51 \pm 0.08$ & $0.53 \pm 0.07$ & $0.55 \pm 0.04$ \\
\hline
\end{tabular}

503

504

Data are presented as a mean $\pm \mathrm{SD}$.

505

506

507

508

509 